\title{
ELEVAGE DE PLANTS FORESTIERS EN MOTTES
}

L'élevage en mottes des plants forestiers (eucalyptus, acacias, caroubiers, arganiers) expérimenté depuis déjà plusieurs années est employé en grand à Agadir depuis I956. C'est dans le cadre du milieu Souss et plus particulièrement Souss côtier qu'il faut situer cet article.

Dans cette technique le plant est élevé dans un pot (motte) obtenu par compression d'un mélange riche en humus, aussi peu riche que possible en argile, qui est ensuite mis en terre avec lui.

\section{Pourquoi?}

- D'abord pour obtenir des plants présentant un meilleur enracinement. Dans le pot classique en terre cuite la racine, sectionnée une ou plusieurs fois au cours du sevrage, tourne et si les pluies tardent un peu donne un véritable chignon qui handicape lourdement le plant.

De ce fait, l'élevage en pots de 9 est à proscrire pour certaines espèces courantes mais dont l'enracinement est particulièrement pivotant: eucalyptus sideroxylon.

Quelles que soient les dimensions des pots, l'élevage d'autres espèces telles que l'arganier et le caroubier est également interdit. La mise en place de plants à racines nues, élevés en pleine terre, étant impossible par suite des conditions climatiques (étages méditerranéens semi-aride et aride), on a cherché un mode d'élevage donnant des plants présentant un enracinement comparable.

- Ensuite, pour doter le plant d'une réserve d'eau. La motte est mise en place après avoir été imbibée d'eau à refus. Cette réserve peut assurer la vie du plant sans apport extérieur pendant environ un mois.

Les colloïdes humiques qui constituent le mélange sont à même ensuite d'absorber et de réserver pour l'alimentation du plant l'eau du sol environnant. 
- La motte contient aussi beaucoup plus de matières nutritives que le pot. Elles sont utilisées par le plant pour son alimentation. Elles lui assurent une croissance très rapide qui permet au jeune arbre de s'installer dans son nouveau milieu en surmontant plus facilement la concurrence de la végétation herbacée.

Suivant la nature de l'enracinement du plant et les conditions du milieu, les dimensions des mottes varient: mottes de 9 centimètres de diamètre et de $\mathrm{I} 7$ centimètres de hauteur, mottes de $\mathrm{I} 2$ centimètres de diamètre et de 22 centimètres de hauteur.

\section{Le matériau}

Le compost utilisé pour la fabrication des mottes doit présenter un certain nombre de qualités:

- être perméable pour que la motte puisse s'imbiber d'une quantité d'eau maximum lors de la plantation.

- se laisser traverser facilement de l'intérieur à l'extérieur par les jeunes racines.

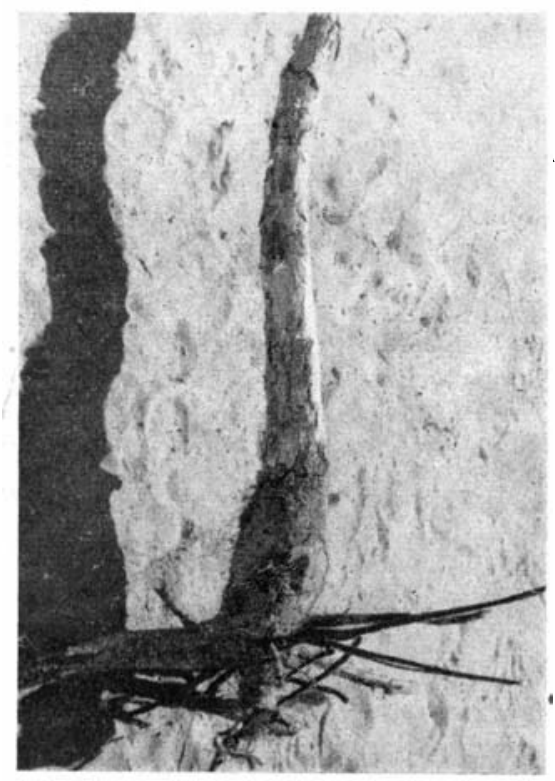

Eucalyptus gomphocephala de $\mathrm{I}_{5}$ ans présentant un enracinement défectueux.

(Cliché Soulères.) 
- donner un mélange suffisamment consistant pour que la motte ne se désagrège pas au cours du transport.

- donner un mélange qui se désagrège le plus facilement possible, une fois la motte mise en place, pour s'incorporer au sol environnant.

Les mottes destinées aux sols argileux peuvent par exemple contenir un pourcentage d'argile plus grand que celles destinées aux sables des dunes où la décomposition est plus lente et difficile.

\section{- être nutritif.}

Après plusieurs expériences, le mélange choisi en fonction des possibilités locales d'approvisionnement est le suivant:

- deux volumes de terreau d'arganier. Il s'agit d'un terreau qui apparaît après la coupe à l'abri des rejets d'arganier et que l'opération de dépressage découvre. Ce terreau est placé dans une fosse cinq ou six mois avant son utilisation et copieusement arrosé pour qu'il puisse se décomposer. Il peut être remplacé par du fumier mélangé au tiers de son volume de sable.

- un volume de terre arable, légèrement argileuse.

- un volume de paille hachée. Elle assure la cohésion de la motte. Les racines en général sortent en en suivant un brin.

Le terreau d'arganier est particulièrement riche en germes animaux et végétaux aui dans un milieu très humide et chand comme celui de la pépinière de Bensergaou pourraient être à l'origine d'une forte mortalité des plants. De nombreux et onéreux traitements préventifs et curatifs sont nécessaires.

Le terreau est d'abord désinfecté au formol !(solution à $2 \%$ de formol commercial à $40 \%$ ). De nombreux germes animaux ou cryptogamiques sont détruits par ce premier traitement. mais le formol est par la suite nuisible aux jeunes plants. La désinfection doit être réalisée au moins un mois avant l'emnloi du terreau. Des essais seront faits l'année prochaine avec le sulfure de carbone qui serait moins toxique pour les semis.

La prévention et la lutte contre les germes cryptogamiaues (Fusarium, Fusicladium, Ö̈dium) s'exercent par la suite orrâce au Karathane WD et au Dithane. Le Karathane est utilisé à raison de 200 grammes par 400 litres de l'eau employée pour le malaxage des trois constituants des mottes.

Chaque semaine les jeunes plants sont arrosés avec une solution analogue jusqu'à l'âge de deux mois. Le traitement s'effectue très tôt 'le matin.

Le Dithane Z. 78. est employé dans les mêmes conditions.

L'H.C.H. en solution (200 grammes d'H.C.H. à $25 \%$ par hectolitre) est utilisé pour le traitement contre les vers blancs. Ces trai- 
tements peuvent paraitre excessifs, mais s'expliquent par l'existence de brumes côtières particulièrement importantes, par le nombre de plants produits (un million en I958) et par le manque de personnel qualifié.

\section{La fabrication des mottes}

Une foịs réalisé à sec le mélange cles trois constituants, celui-ci est malaxé comme du mortier après avoir été imbibé d'eau jus-. qu'à ce qu'il devienne parfaitement homogène.
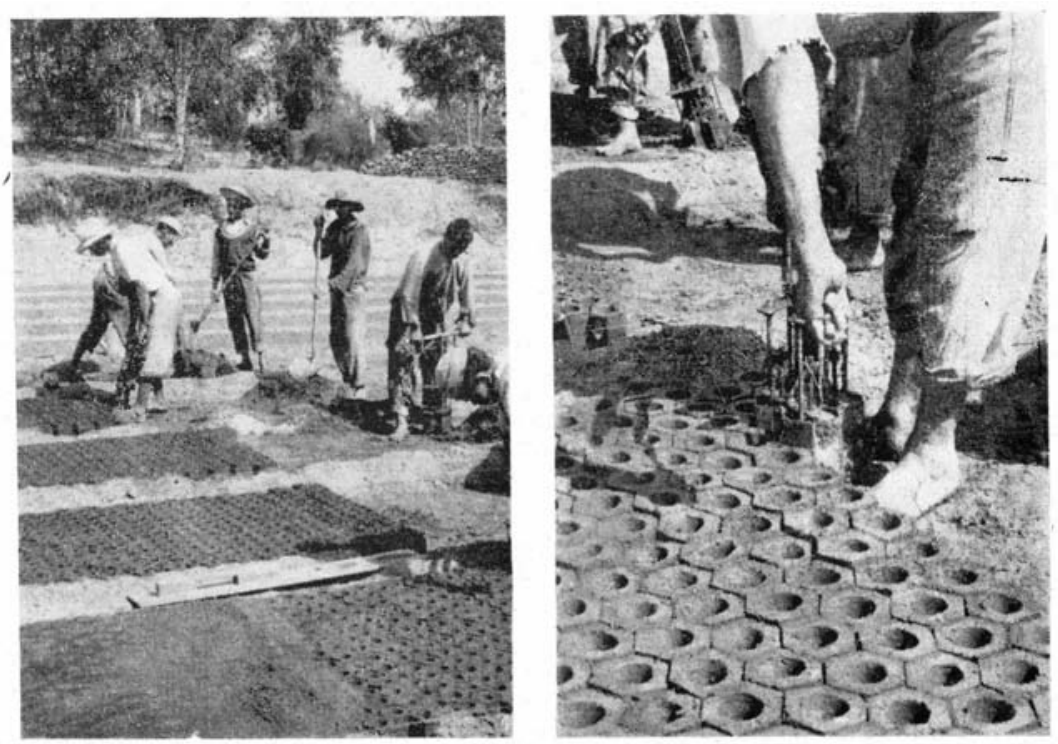

Pépinière de Bensergaou.

Fabrication des mottes.

(Clichés Soulères.)

Les mottes sont ensuite confectionnées avec des presses à mottes achetées dans le commerce (pour l'élevage des orangers), fabriquées suivant un modèle en usage à Casablanca ou par un artisan local pour celles donnant les mottes du plus gros modèle. Chaque machine produit un seul type de mottes par deux ou quatre exemplaires à la fois.

Les meilleurs résultats ont jusqu'à maintenant été obtenus avec le type le plus rustique.

Ces machines permettent toutes de placer les mottes au fur et à mesure de leur fabrication dans des planches de I,20 mètre de lar- 
geur, préparées à l'avance et séparées l'une de l'autre par des allées de 0,50 mètre environ.

Le fond de ces planches est à une profondeur telle que la partie supérieure des mottes soit à peu près au même niveau que les chemins. Ceux-ci s'enfoncent légèrement ensuite sous l'effet du tassement.

\section{Semis et repiquage - Elevage}

Les alvéoles des différentes mottes placées les unes à côté des autres sont remplies d'un mélange de terreau d'arganier et de terre franche analogue à celui utilisé dans les pots de terre cuite.

Ce mélange est légèrement comprimé à la main dans la partie centrale. C'est là que les graines sont semées puis légèrement enterrées sous quelques millimètres de sable. A une des exprémités de la planche sont placés de gros pots de terre cuite dans lesquels est également effectué un semis. Les jeunes plants en provenant seront utilisés pour les repiquages.

Les mottes et les pots sont ensuite copieusement arrosés et recouverts d'une ombrière basse $\left(\mathrm{I}_{5} \mathrm{~cm}\right)$ qui les abritera pendant une quinzaine de jours.

Un deuxième semis peut alors être effectué dans les mottes où rien n'est apparu, mais il n'est pas conseillé de procéder à un troisième semis, car l'on obtiendrait des planches trop hétérogènes.

Quinze jours après le deuxième semis, les jeunes plants, de 4 à 6 feuilles environ. obtenus dans les pots de terre cuite sont utilisés pour les repiquages dans les mottes où aucun semis ne s'est développé.

Pour ce faire, on vide l'alvéole au moyen d'une cuillère en raclant bien les parois, on creuse légèrement le fond avec un plantoir et on arrose copieusement.

La motte s'est en effet desséchée et ne se laisserait pas traverser sans cela par les racines.

Le jeune plant est mis en place, bien vertical. La cavité est remplie tout autour de lui avec du compost. La planche est ensuite bien arrosée et recouverte d'une ombrière qui pourra être enlevée au bout d'une semaine.

L'élevage des plants par la suite ne présente que de faibles différences avec l'élevage classique en poterie de terre cuite. Les planches sont recouvertes d'une mince couche de sable qui maintient une humidité constante et supprime les solutions de continuité entre les mottes.

Il n'y a pas de sevrage avec les eucalyptus et les acacias. Cette opération reste cependant quelquefois nécessaire pour les arganiers et les caroubiers. 
Ceux-ci sont élevés dans les plus grosses mottes qui s'enlèvent et se remettent en place facilement.

L'arrosage des plants doit être beaucoup moins abondant, surtout vers la fin de la campagne de pépinière, que pour les plants élevés en pots.

Quand l'eau est chargée en sel, les excès d'arrosage sont particulièrement nuisibles. Le sel en effet se concentre dans la motte, cause un dépérissement du plant et le sensibilise aux attaques des champignons.

En I957, près de 200000 Eucalyptus gomphocephala élevés en mottes n'ont pas été arrosés du $\mathrm{I}^{\text {er }}$ octobre à leur plantation en décembre. Ils ont seulement reçu à plusieurs reprises de petites ondées.

De même, des plants d'Acacia cyclops n'ont commencé à se développer qu'après une réduction sensible de l'arrosage.

\section{Transport et plantation}

Cinq ou six mois après le semis, les jeunes plants d'eucalyptus sont bons à planter. Si les conditions sont favorables, les mottes sont extraites rles planches et placées dans des caisses confectionnées spécialement, 30 dans chaque, après que les jeunes plants aient été fortement rabattus (3 à 4 feuilles) et que leurs racines aient été coupées au ras de la motte.

Ces jeunes plants ne doivent pas être arrosés auparavant, contrairement à ce qui se passe avec les plants en pots. pour que la motte ne se désagrège pas au cours du transport.

Les caisses sont transportées par camion ou à dos d'animaux jusqu'aux lieux de plantation où elles sont plongées dans des bacs remplis d'eau.

D'abondantes bulles apparaissent en surface. Les caisses sont retirées du bac dès que le bouillonnement cesse. Les mottes sont alors pleines d'eau et peuvent être mises en place.

Quand le sol est très meuble (sable) elles doivent être enterrées profondément car le vent en les déchaussant les fait durcir. Les mottes ne peuvent plus se désagréger et le plant meuit.

\section{Prix de revient}

Le rendement est évidemment fonction de la qualification de l'ouvrier employé à la fabrication des mottes et aussi du pourcentage de réussite.

Un ouvrier entraîné et régulièrement alimenté en compost peut fabriquer I 600 à I 800 mottes de 9 centimètres de diamètre par jour, ce qui représente environ $\mathrm{t} \mathrm{m}^{3} \mathrm{~d}$ u mélange utilisé. 
Après le semis: les ombrières basses et les pots de terre cuite où pousseront les plants destinés à être repiqués.
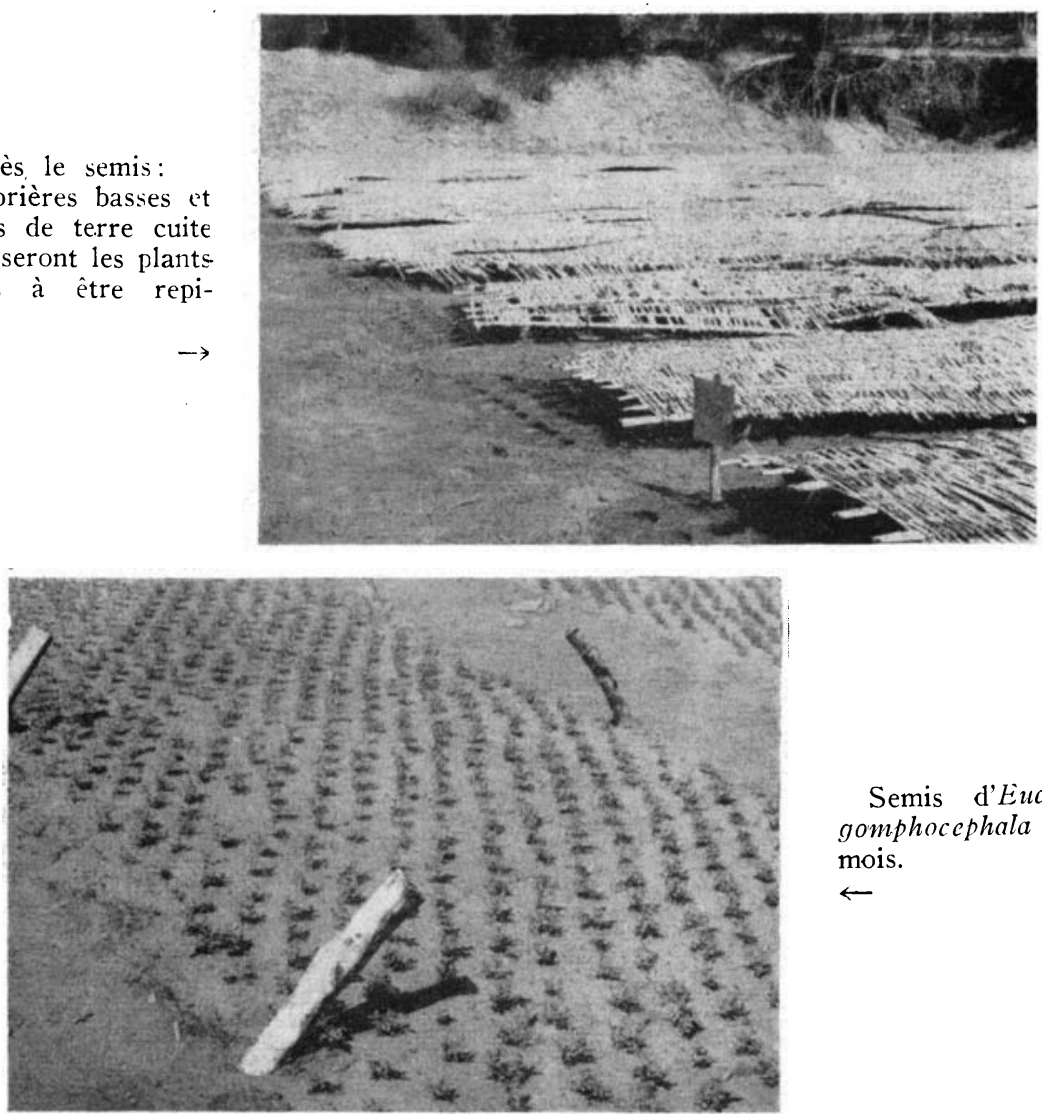

Semis d'Eucalyptus gomphocephala de un mois.

Plants de 5 mois élevés en mottes.

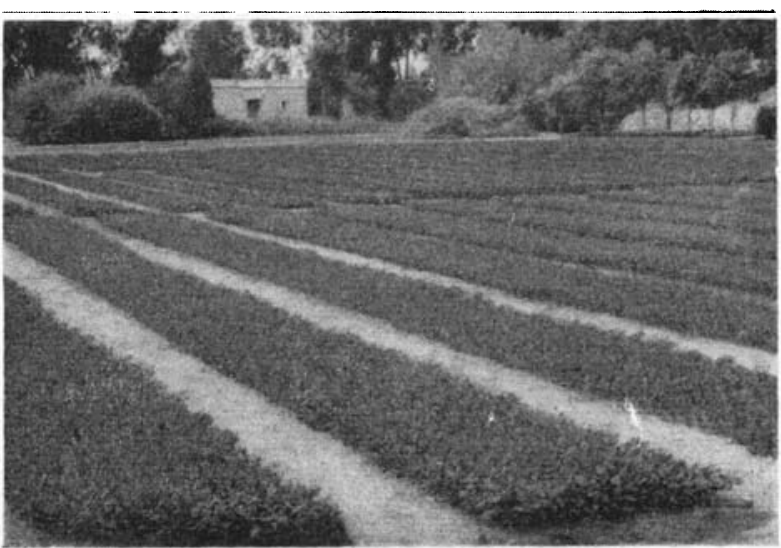

Pépinière de Bensergaou.

$\therefore$ (Clichés - Soulères.) 


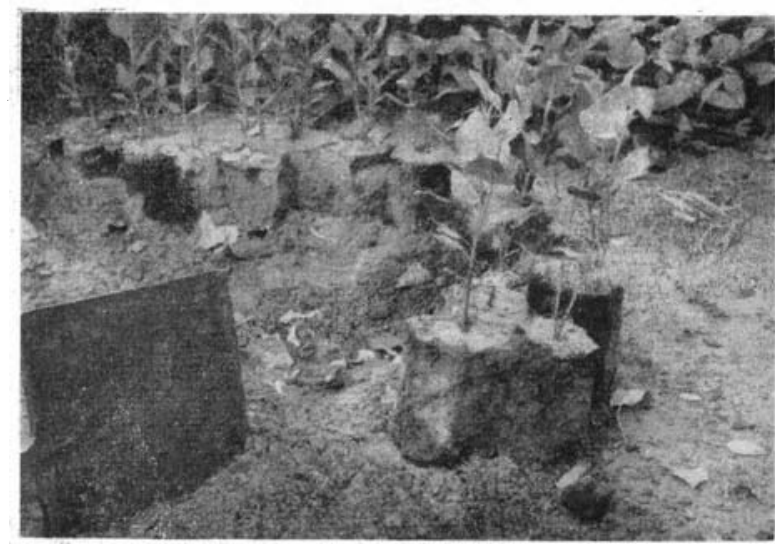

Plants d'Eucalyptus gomphocephala de 5 mois extraits des planches.

Enracinement d'un plant d'Eucalyptus gomphocephala de 5 mois élevé en motte.
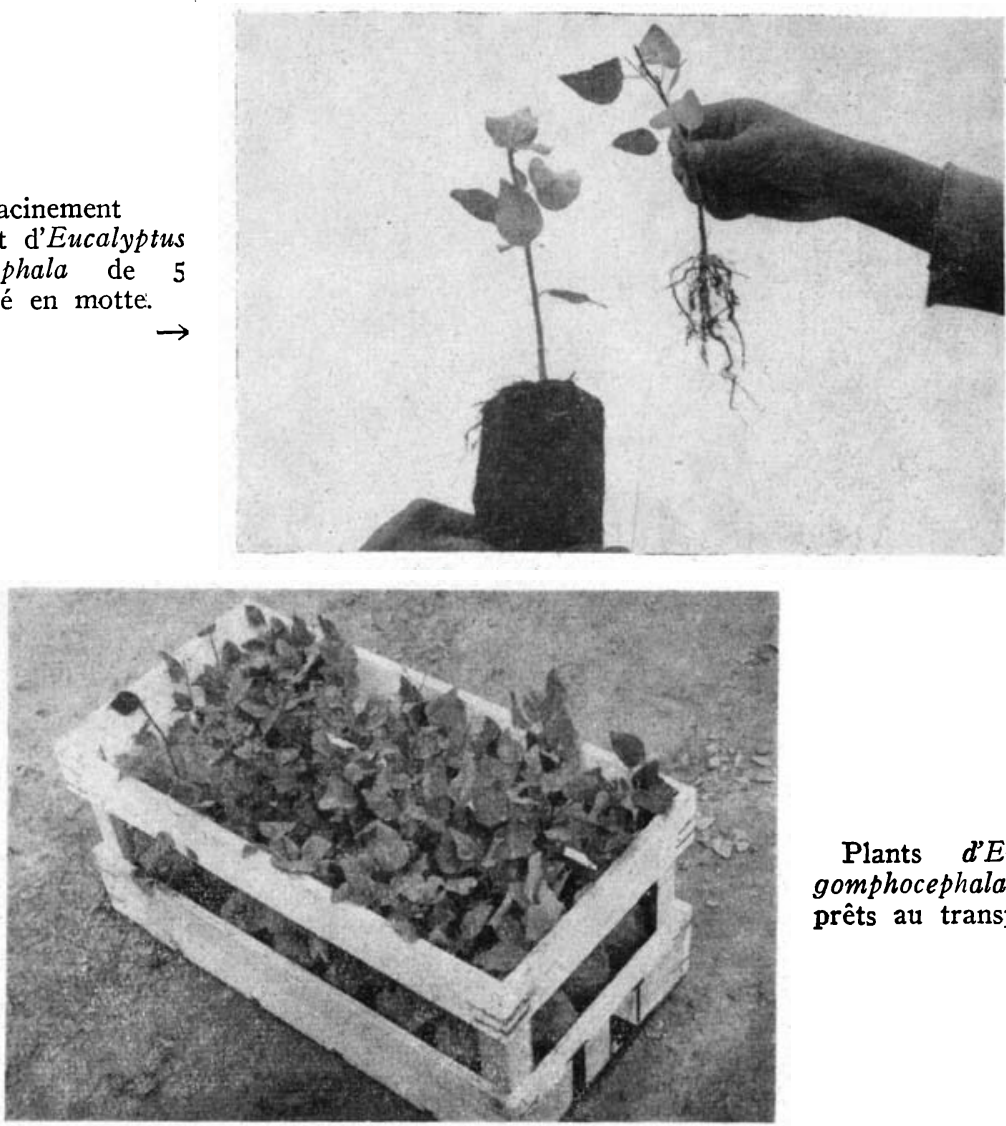

Plants d'Eucalyptus gomphocephala (5 mois) prêts au transport.

Pépinière de Bensergaou.

(Clichés Soulères.) 
En 1957, les éléments du prix de revient ont été les suivants pour r ooo mottes de $9\left(0,66 \mathrm{~m}^{3}\right)$ sans tenir compte des frais généraux de fonctionnement de la pépinière.

\section{Fournitures:}

- terreau (achat et transport) $\ldots \ldots \ldots \ldots \ldots$ I $400 \mathrm{~F}$

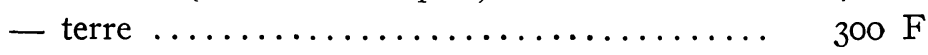

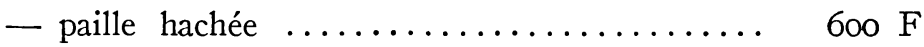

Main-d'œuvre (le salaire journalier d'un ouvrier qualifié est de 450 francs environ).

- préparation du compost .............. $800 \mathrm{~F}$

- mise en place des mottes .............. $400 \mathrm{~F}$

- traitements antiparasite $\ldots . \ldots \ldots \ldots \ldots$. I00 $\mathrm{F}$

I $300 \mathrm{~F}$

La motte prête à être semée reviendrait donc à 3,6 francs. Cela n'est toutefois pas le prix de revient réel, car l'on n'obtient pas un plant par motte.

En 1957, le pourcentage de réussite a été de $80 \%$. De ce fait, la part revenant à la motte dans la dépense due à la production d'un plant s'élève à 4,5 francs.

Le prix de revient d'un plant élevé en motte est donc assez nettement supérieur à celui d'un plant élevé en pot. La différence entre ces deux prix de revient tendra d'ailleurs à diminuer au fur et à mesure que les techniques d'élevage se perfectionneront et que la lutte anticryptogamique s'améliorera.

Par contre, les plants élevés en mottes ont un pourcentage de reprise supérieur et une croissance plus rapide et homogène. Ils surmontent beaucoup plus facilement la concurrence de la végétation voisine.

Pour ces raisons, le prix de revient de l'arbre vivant et bien venant, après deux années de plantation ${ }_{x}$ est nettement moins élevé avec ce système.

Lors d'une année particulièrement sèche, le reboisement n'a été possible que grâce à la technique d'élevage des plants en mottes.

Au cours de l'hiver 1956-1957, la forêt des dunes d'Agadir, rive gauche de l'oued Souss, n'a reçu que 56,6 millimètres de pluie (pluviométrie relevée au poste forestier de Rokein du $\mathrm{I}^{\text {er }}$ octobre 
r956 au 30 septembre I957). Le pourcentage de réussite pour les 50 ooo Eucalyptus gomphocephala élevés en mottes mis en place a été de $42,3 \%$.

Leur reprise et leur croissance ont été, il est vrai, favorisées par d'abondants brouillards côtiers.

G. Soulères,

Ingénieur des Eaux et Forêts à Agadir.

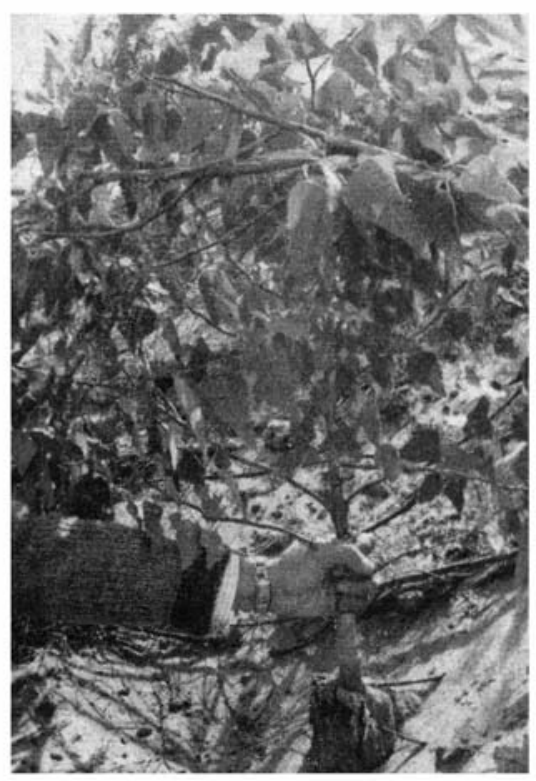

Jeune Eucalyptus gomphocephala 7 mois après sa mise en place.

(Cliché Soulères.) 